\title{
Within-host diversity of SARS-CoV-2 in COVID-19 patients with variable disease severities
}

\author{
Hebah A. Al-Khatib, Fatiha Benslimane, Israa E. Elbashir, Asmaa A. Althani, Hadi M. Yassine* \\ Biomedical Research Center, Qatar University, PO Box 2713, Doha, QATAR \\ *Corresponding Author
}

\section{BACKGROUND}

The ongoing pandemic of SARS-COV-2 has already infected millions of people worldwide. The majority of COVID-19 patients are either asymptomatic or have mild symptoms. Yet, about $15 \%$ of the cases experience severe complications and require intensive care. Factors determining disease severity are not yet fully characterized. The within-host diversity and their relation to severity were reported in infected patients during SARS-CoV-1 and MERS coronaviruses outbreaks; however, little is known about their effect on virus evolution, transmissibility and pathogenies. To date, thousands of SARS-CoV-2 sequences have been deposited in public databases; yet, we still lack fundamental information about the within-host diversity of SARS-CoV-2 and its possible role in disease severity.

\section{OBJECTIVE}

The aim of this study is to compare the SARS-CoV-2 diversity in COVID-19 patients with mild and severe manifestations and investigate its impact on clinical disease severity.

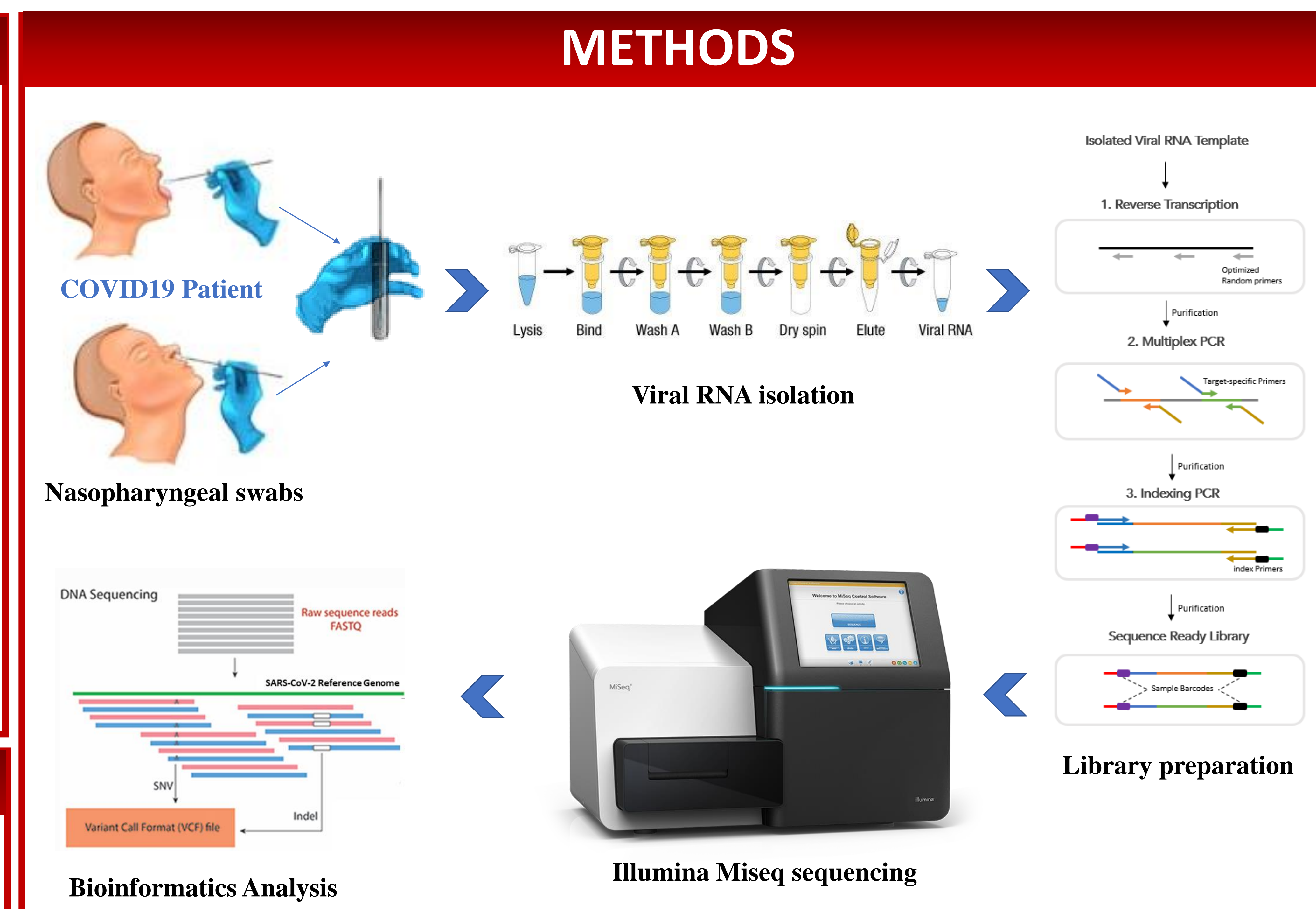

Bioinformatics Analysis

\section{RESULTS}

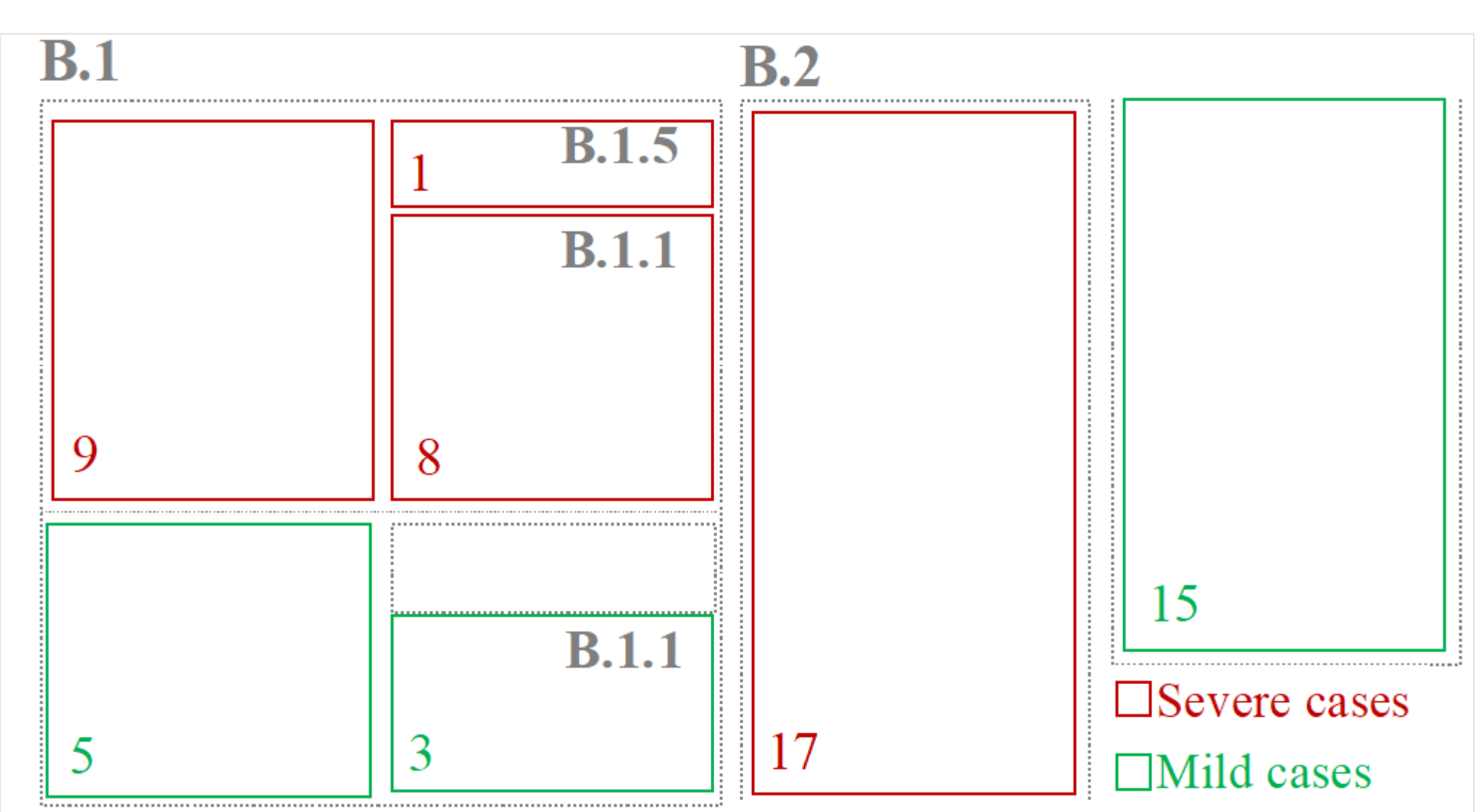

SARS-CoV-2 Lineage: Lineage are based on the nomenclature system proposed in Rambaut et al., 2020. Numbers inside the boxes indicates the number of samples within each lineage.

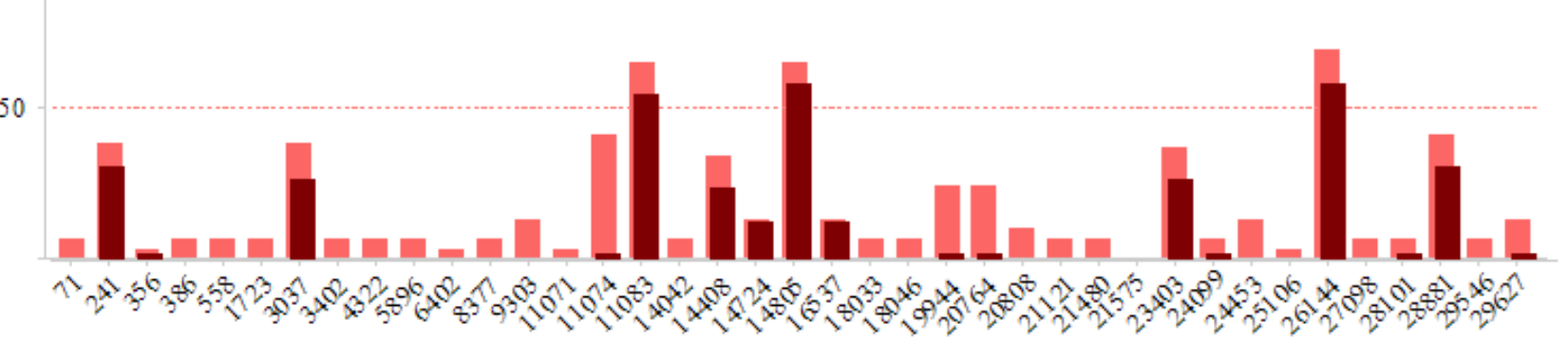

$\infty_{\text {vild smproms }}$

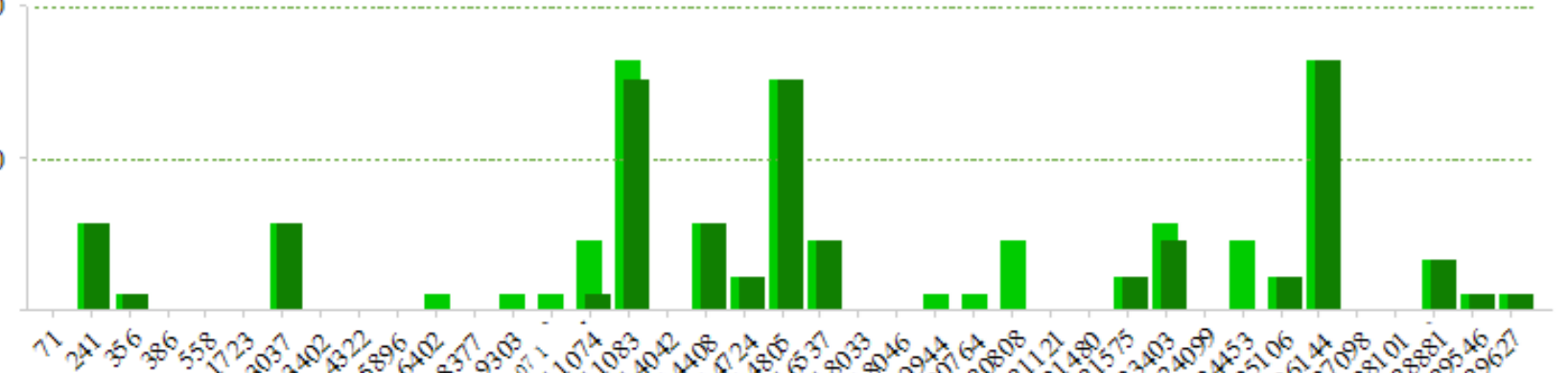

Comparison of variants found in mild cases (green) and severe cases (red). Colored parts of the circles (on the left) represent variants seen in more than one patient. Only variants found in more than one patients were used to generate bar charts. Bar charts demonstrate the prevalence of each variant in patients within each group (severe vs mild). Bars with darker colors demonstrate variants detected at the consensus sequence level.

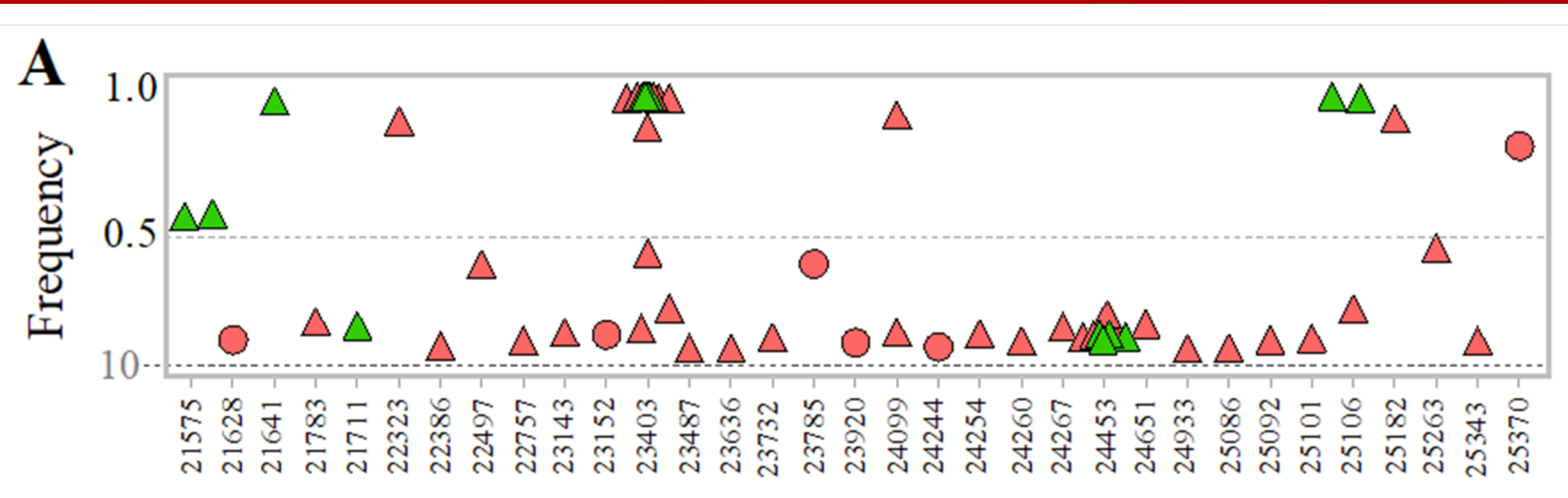

B

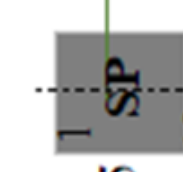

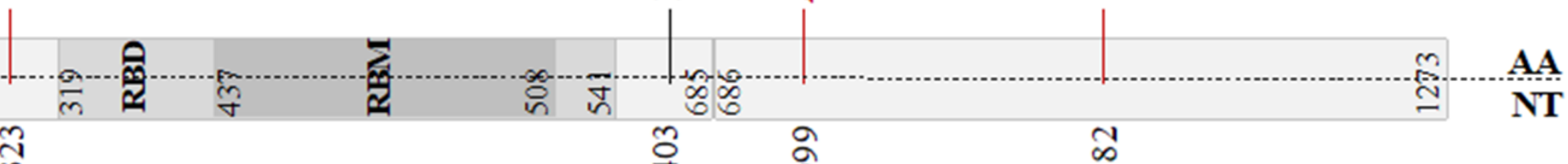

Variants found in the SARS-CoV-2 spike glycoprotein. (A) Genomic position and frequency of $S$ variants detected in al patients. (B) Schematic representation showing consensus mutations found in mild (green) and severe (red) cases. D614G mutation was found in both mild and severe cases.

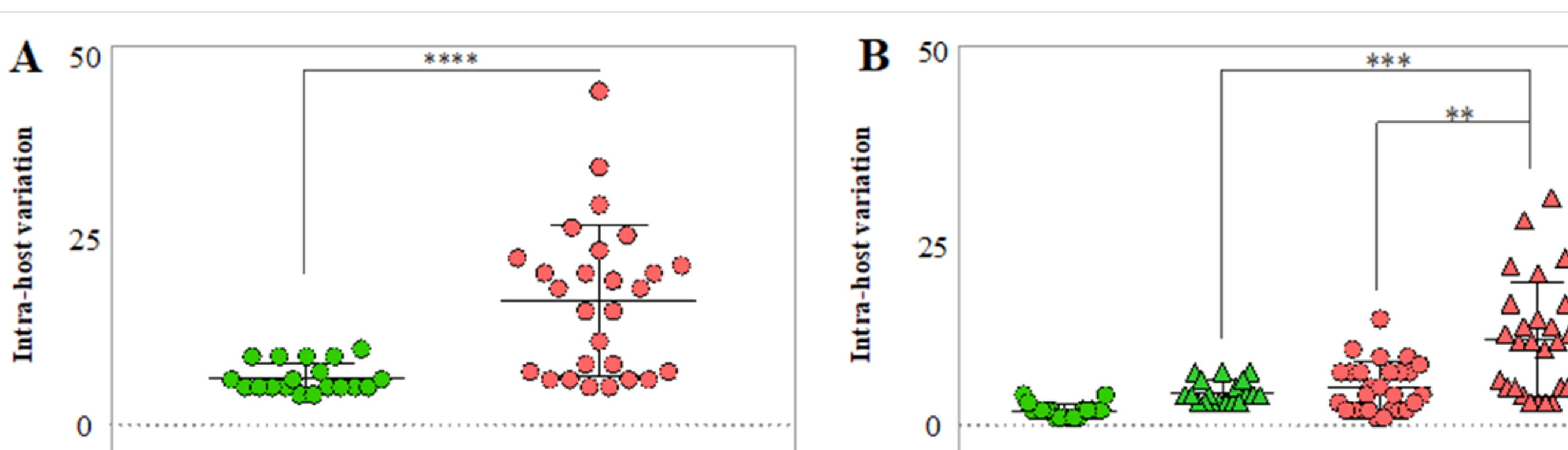

Assessment of within-host SARS-CoV-2 diversity. (A) Tota number of variants detected in each patient with mild (green) and severe (red) symptoms. (B) Comparsion of number of synonymous (S) and non-synonymous (NS) mutations found withih each patinet of the two groups (mild vs severe cases). Pvalues are indicated as follows: $* * * *$ for $\mathrm{p}$-value less than $0.0001, * * *$ for $p$-value less than 0.001

\section{CONCLUSION}

Exploring within-host diversity of this newly emerged virus, SARS-CoV-2, has revealed significant differences between mild and severe cases, particularly among older patients. Therefore, further investigation of within-host diversity role in disease severity is of significance at this stage of the pandemic and should be considered in future studies. It is also crucial to compare viral quasispecies in patients of different age groups as well as from different sample types. This would also help us better understand disease severity

\section{ACKNOWLEDGEMENT}

This work was supported by Qatar University under internal grant (QUCG-BRC-20/21-1) and Qatar National Research Fund grant under grant (RRC-2-039). We acknowledge the support from the Ministry of Public Health, Doha, Qatar and the virology laboratory in Hamad Medical Corporation, Doha, Qatar for their efforts in the preparation of nasopharyngeal samples and collection of clinical data.

\section{REFERENCES}

Cui, J., F. Li, and Z.L. Shi, Or 